\title{
Phase behaviour of mixtures of polyethylene glycol and polypropylene glycol: Influence of hydrogen bond clusters on critical composition fluctuations
}

\author{
Susanne Eckert-Kastner, ${ }^{a}$ Gerhard Meier $^{b}$ and Ingo Alig*a \\ a Deutsches Kunststoff-Institut, D-64289 Darmstadt, Schloßgartenstr. 6, Germany \\ b IFF "Weiche Materie”, FZ-Jülich, Postfach 1913, D-52425 Jülich, Germany
}

Received 1st May 2003, Accepted 9th June 2003

First published as an Advance Article on the web 26th June 2003

\begin{abstract}
The critical behaviour of a binary polymer blend of polyethylene glycol 600 (PEG600) and polypropylene glycol 1000 (PPG1000) was investigated by static and dynamic light scattering. The measurements were carried out in the homogeneous one-phase region (above the critical temperature $T_{\mathrm{c}}$ ) and in the two-phase region in both coexisting phases. Additionally to the critical composition ( $y_{\mathrm{PPG}}=0.46, y_{\mathrm{PPG}}$ : mass fraction of PPG), a mixture of non-critical composition $\left(y_{\mathrm{PPG}}=0.365\right)$ was investigated in the one- and two-phase region. From the light scattering measurements, the critical exponents and the amplitudes of the correlation length $\xi$ and the mutual diffusion coefficient $D$ were determined. For the critical mixture the results for the critical exponents and amplitudes of $\xi$ in the one- and two-phase regions are in accordance with the predictions of the 3d-Ising model. The measurements of $D$ in the two-phase region in a wider temperature range gave an indication for a crossover from Ising to mean field behaviour. Since the low molar mass of the PEG and PEG oligomers would lead to Ising behaviour in the composition and temperature range studied, this crossover has to be attributed to an increase of the effective molar mass caused by clusters built by intermolecular hydrogen bonds. The assumption of these clusters is supported by static light scattering and the phase diagram. The data of the non-critical mixture could be described in the frame of the pseudospinodal concept. The results for the non-critical mixture support the interpretation of the data of the critical mixture.
\end{abstract}

\section{Introduction}

Critical phenomena of polymer blends in the one-phase region have been studied extensively by scattering methods, e.g. by static and dynamic light scattering experiments (see e.g. ${ }^{1-5}$ ). The static light scattering experiments yield the critical exponents and amplitudes for the correlation length and for the susceptibility, whereas dynamic light scattering experiments can be applied to study the dynamical critical behaviour.

In literature there are described far less corresponding examinations on binary mixtures in the two-phase region than in the one-phase region. For the three-dimensional (3d) Ising model, renormalization group calculations predict the same values of the critical exponents in the one- and in the two-phase region of the phase diagram. ${ }^{6}$ This has been confirmed so far for low molecular mixtures where light scattering experiments have been carried out in the coexisting phases of the two-phase region. ${ }^{7-10}$ On the other hand, to our knowledge, no systematic light scattering experiments in the two-phase region of polymer mixtures in real equilibrium have been published so far. The correlation length of polymer mixtures in the two-phase region has been measured by small-angle neutron scattering (SANS), ${ }^{11}$ and no measurements of the dynamical critical properties are available. An additional drawback comes from the fact that the experiments described in ${ }^{11}$ were not carried out in thermodynamic equilibrium of the two coexisting phases.

Furthermore, systematic investigations of the critical composition fluctuations of non-critical mixtures are rare. There are light scattering measurements of non-critical low molecular mixtures ${ }^{9,10,12-14}$ but, to our knowledge, there are no scattering experiments on polymer mixtures of non-critical concentrations where a crossover from Ising to mean field behaviour was observed.

Here, we report on measurements of static and dynamic light scattering on an oligomer blend of PEG600/PPG1000 at the critical composition $\left(y_{\mathrm{PPG}, \mathrm{c}}=0.46\right)$, with a molar mass of $M=600 \mathrm{~g} \mathrm{~mol}^{-1}$ for the PEG and $M=1000 \mathrm{~g} \mathrm{~mol}^{-1}$ for the PPG and small molar mass distributions for both samples $\left(M_{\mathrm{w}} / M_{\mathrm{N}} \approx 1.03\right)$. The degrees of polymerisation of PEG600 and PPG1000 are $N_{\mathrm{PEG}}=14$ and $N_{\mathrm{PPG}}=18$. Additionally, a mixture of non-critical composition $\left(y_{\mathrm{PPG}}=0.365\right)$ was investigated in the homogeneous one-phase region and in the two-phase region in both coexisting phases. To measure in both coexisting phases separately, we had to wait typically between one and two weeks until the phases were macroscopically separated and no further change in the scattered intensity could be detected.

One of the aims of this work were measurements of the correlation length and the diffusion coefficient in the one-phase region and also in both coexisting phases, i.e. on the binodal curve, at the final stage of phase separation, in order to prove the theoretical prediction ${ }^{6}$ of identical critical exponents below and above the critical point for a polymeric system. Therefore the values of the critical exponents for the correlation length and the collective diffusion coefficient were determined by the static and dynamic light scattering data of the PEG//PPG mixtures.

Generally one may expect a crossover from 3d-Ising to mean field behaviour in polymer blends depending on chain length. ${ }^{15,16}$ This crossover is governed by the Ginzburg criterion. ${ }^{17}$ The Ginzburg number can be defined as the reduced temperature where the crossover occurs $\left(\mathrm{Gi} \equiv \varepsilon_{\mathrm{Gi}}=\right.$ $\left|T_{\mathrm{Gi}}-T_{\mathrm{c}}\right| / T_{\mathrm{c}}, T_{\mathrm{c}}$ : critical temperature). For low molecular 
liquids, an Ornstein-Zernike model based on the susceptibility leads to values of $0.01<\mathrm{Gi}<0.04$ for the Ginzburg number ${ }^{18}$ whereas for polymer mixtures, the Ginzburg number is dependent on the degree of polymerisation. For symmetric polymer mixtures with a degree of polymerisation $N$, the Ginzburg number is inversely proportional to $N .^{15,16}$

In a preceding paper ${ }^{19}$ we had thoroughly investigated the influence of hydrogen bonds on the phase diagram of PEG/ PPG mixtures. There we found clusters built by intermolecular hydrogen bonds leading to a higher "effective molar mass" and a larger "effective polydispersity". The radius of gyration $R_{\mathrm{g}, \mathrm{cluster}}$ of this clusters measured by static and dynamic light scattering was found to be in the order of 250 to $550 \mathrm{~nm}$. Therefore it seems very interesting to view upon that with regard to the molar mass dependence of the Ginzburg number. Although the low molar mass of the PEG and PPG oligomers would lead to Ising exponents in the temperature range studied, for the large "effective molar mass" of the hydrogen bond clusters a crossover to mean field behaviour for the diffusion coefficient is expected.

The paper is organised as follows: After a survey of the theory for light scattering of critical mixtures (Sections II A and B), the extension to non-critical mixtures is described in Section II C. In Section III, a sketch of the experimental conditions is given. The phase diagram of the system is shown in Section IV A, the analysis of the static and dynamic light scattering data is described in Section IV B (critical mixture) and IV C (non-critical mixture). The paper is closed by some concluding remarks.

\section{Theory}

\section{A. Static light scattering}

In the vicinity of the critical point of demixing, the scattered light intensity $I(\boldsymbol{q})$ of a critical binary polymer mixture mainly results from fluctuations of the concentration. This intensity $I(\boldsymbol{q})$ is proportional to the structure factor $S(\boldsymbol{q})$, the Fourier transformation of the two point correlation function $G(\boldsymbol{r})$ :

$$
S(\boldsymbol{q})=V^{-1} \int_{V} \mathrm{~d}^{3} \boldsymbol{r} G(\boldsymbol{r}) \exp (\mathrm{i} \boldsymbol{q} \boldsymbol{r}),
$$

where $V$ is the scattering volume, and $G(\boldsymbol{r})$ is given by:

$$
G(\boldsymbol{r})=G\left(\boldsymbol{r}_{1}-\boldsymbol{r}_{2}\right)=\left\langle\phi\left(\boldsymbol{r}_{1}\right) \phi\left(\boldsymbol{r}_{2}\right)\right\rangle-\langle\phi\rangle^{2}
$$

which measures the correlation between the concentration $\phi\left(\boldsymbol{r}_{1}\right)$ and $\phi\left(\boldsymbol{r}_{2}\right)$ at two points $\boldsymbol{r}_{1}$ and $\boldsymbol{r}_{2}, \phi=\phi_{\mathrm{B}}=1-\phi_{\mathrm{A}}$, where $\mathrm{A}$ and $\mathrm{B}$ are the polymer species.

If $q \xi$ is not too large, it is approximately correct to write an Ornstein-Zernike form for $G(\boldsymbol{r}):^{20}$

$$
G(\boldsymbol{r}) \sim \frac{\exp (-r / \xi)}{r}
$$

In eqn. (3) the correlation length $\xi$ is introduced. By explicitly solving eqn. (1) with $G(\boldsymbol{r})$ given by eqn. (3), one gets the Ornstein-Zernike scattering law

$$
S(\boldsymbol{q})=S(\boldsymbol{q}=0)\left(1+q^{2} \xi^{2}\right)^{-1} .
$$

The temperature dependence of the correlation length $\xi$ is given by

$$
\xi=\xi_{0} \varepsilon^{-\nu},
$$

with the critical exponent $\nu$ of the correlation length and the reduced temperature $\varepsilon=\left(T-T_{\mathrm{c}}\right) / T_{\mathrm{c}}(\nu=0.63$ for the $3 \mathrm{~d}$ Ising behaviour and 0.5 for the mean field model).

In the two-phase region the temperature dependence of $\xi$ is given by

$$
\xi=\xi_{0}^{-}|\varepsilon|^{-\nu_{-}}
$$

According to renormalization group calculations for the $3 \mathrm{~d}-$ Ising behaviour, ${ }^{6}$ the critical exponents above and below $T_{\mathrm{c}}$ are the same $\left(\nu=\nu_{-}\right)$and the relation between the critical amplitudes is universal. The ratio between the amplitudes of the correlation length is $\left(\xi_{0}^{-} / \xi_{0}\right)_{\text {Ising }}=0.51 \pm 0.003 .{ }^{21}$ In the mean field region, $\nu=\nu_{-}$and $\left(\xi_{0}^{-} / \xi_{0}\right)_{\mathrm{mf}}=1 / \sqrt{ } 2$.

\section{B. Dynamic light scattering}

In a binary mixture, the central quasielastic component in the spectrum of scattered light is caused by the diffusive decay of concentration fluctuations. By measuring the time autocorrelation function of the scattered light intensity, the dynamic structure factor $S(\boldsymbol{q}, t)=\langle\delta \phi(\boldsymbol{q}, t) \delta \phi(-\boldsymbol{q}, 0)\rangle$ with $\delta \phi(\boldsymbol{q}, t)=$ $\phi(\boldsymbol{q}, t)-\langle\phi\rangle$, the decay of the order parameter $\delta \phi$ can be obtained. For a single exponential decay of the dynamic structure factor, the decay rate $\Gamma(\boldsymbol{q})$ is defined by

$$
S(\boldsymbol{q}, t)=S(\boldsymbol{q}) \exp (-\Gamma(\boldsymbol{q}) t)=S(\boldsymbol{q}) \exp \left(-D(\boldsymbol{q}) q^{2} t\right),
$$

where $S(\boldsymbol{q})$ is the static structure factor and $D(\boldsymbol{q})$ is the collective diffusion coefficient.

The measured decay rate contains, beside the critical part, a non-critical background. This is commonly described by splitting $D(\boldsymbol{q})$ into a background term $D_{\mathrm{b}}$ which is assumed to be constant, and the critical term $D_{\mathrm{c}}(\boldsymbol{q}): 22,23 D(\boldsymbol{q})=D_{\mathrm{b}}+D_{\mathrm{c}}(\boldsymbol{q})$.

The expression of the critical term $D_{\mathrm{c}}(\boldsymbol{q})$ from mode coupling theory is: ${ }^{24}$

$$
\begin{gathered}
D_{\mathrm{c}}(\boldsymbol{q})=\frac{\Gamma_{\mathrm{c}}(\boldsymbol{q})}{q^{2}}=R \frac{k_{\mathrm{B}} T}{6 \pi \eta_{\mathrm{S}} \xi} K(x), \\
K(x)=\frac{3}{4 x^{2}}\left[1+x^{2}+\left(x^{3}-x^{-1}\right) \arctan x\right],
\end{gathered}
$$

with $\eta_{\mathrm{S}}$ being the shear viscosity, $x=q \xi, K(x)$ the Kawasaki function, and $R$ a universal dynamic amplitude ratio $(R=$ 1.056 for $T=T_{\mathrm{c}}{ }^{25}$ ).

Already in the hydrodynamic region $(q \xi \ll 1)$ where most of the data were taken, the measured diffusion coefficient $D(\boldsymbol{q})$ is given by $D_{\mathrm{c}}(\boldsymbol{q})$ with

$$
\lim _{q \xi \rightarrow 0} D_{\mathrm{c}}=R \frac{k_{\mathrm{B}} T}{6 \pi \eta \xi},
$$

and thus contributions of the background can be safely neglected. ${ }^{1}$ The temperature dependence of the diffusion coefficient in the hydrodynamic limit is given by

$$
D_{\mathrm{c}}=D_{0} \varepsilon^{\nu *}
$$

where $\nu^{*}$ is the critical exponent of the diffusion coefficient: $\nu^{*}=\nu\left(1+x_{\eta}\right)$, with $x_{\eta}=0$ for the mean field model; for the 3d-Ising model $x_{\eta}=0.054$ from a mode coupling approach ${ }^{26}$ and 0.065 from renormalization group theory. ${ }^{27}$ This results in a value of $\nu^{*}=0.5$ in the mean field case, and $\nu^{*}=$ $0.664^{26}$ respectively $\nu^{*}=0.671^{27}$ in the $3 \mathrm{~d}$-Ising case.

In the two-phase region, the temperature dependence of the diffusion coefficient is given by

$$
D=D_{0}|\varepsilon|^{\nu_{-}^{*}}
$$

with $\nu_{-}^{*}=\nu^{*}$.

\section{Extension to non-critical mixtures}

The data of the non-critical mixture are analysed by the pseudospinodal concept in this paper. This concept was first used by Benedek ${ }^{28}$ for the description of the data at non-critical isochores of $\mathrm{SF}_{6}$ and was extended to non-critical binary mixtures by Chu et al. ${ }^{12}$ The basic assumption of the pseudospinodal concept is that the temperature dependence of the thermodynamic and transport properties of a system at non-critical isochores can be described with similar power laws as at the 
critical isochore. It is assumed that at non-critical isochores these properties diverge at a pseudospinodal temperature $T_{\mathrm{PS}}$. The pseudospinodal temperature $T_{\mathrm{PS}}$ is an empirical parameter and can be determined by a fit of a power law to the data. Then, the correlation length of a non-critical mixture is assumed to obey to the following equation:

$$
\xi=\tilde{\xi}_{0}\left(\frac{T-T_{\mathrm{PS}}}{T_{\mathrm{PS}}}\right)^{-\tilde{\nu}},
$$

Similar equations are valid for the dynamical properties like for example the diffusion coefficient. It has to be noticed here that the pseudospinodal concept is fully empirical. Without measurements in the metastable state it is not possible to determine whether the pseudospinodal temperature is identical to the spinodal temperature which separates the metastable from the unstable region of the phase diagram. However, the gasliquid transition of one-component systems ${ }^{28}$ and the phase behaviour of low molecular binary mixtures ${ }^{9,10,12-14,29,30}$ can be described satisfactorily in the frame of the pseudospinodal concept. Based on these results we have assumed this concept to be also valid for the data on non-critical polymer blends. This assumption is justified by the universality hypothesis of critical phenomena which states that the results do not depend on structural details of the materials used.

An alternative concept of data reduction for the scaling of the correlation length is the application of parametric equations of state introduced by Schofield ${ }^{31}$ and Josephson. ${ }^{32}$ For the parameter transformation, the knowledge of the coexistence curve of the mixture is essential. Since in our case the coexistence curves of the critical and the non-critical mixture are different from each other (see Sec. IV A), an analysis of the light scattering data in the frame of the model of parametric equations of state is not possible. Therefore the data could only be analysed in the frame of the pseudospinodal concept.

The description of the crossover from Ising to mean field behaviour by the Ginzburg number Gi uses the reduced temperature as a measure for the distance from the critical point. But the reduced temperature is only a good measure for a critical mixture in the one-phase region. For the examination of other locations of the phase diagram (e.g. mixtures of non-critical composition) the susceptibility or the correlation length are more suitable parameters in order to measure the distance from the critical point. ${ }^{18}$ For polymer mixtures, the Ginzburg criterion for the correlation length was determined depending on the radius of gyration $R_{\mathrm{g}}$ and the number of the statistical segments $N_{a}$ as: $:^{33}$

$$
\xi_{\mathrm{Gi}}=R_{\mathrm{g}} \sqrt{N_{a}},
$$

with $R_{\mathrm{g}}=a\left(N_{a} / 6\right)^{1 / 2}$ and $a$ the length of a statistical segment.

With eqn. (13) and the assumption of the pseudospinodal concept (see eqn. (12)) it is possible to describe the transition from mean field to Ising behaviour also for non-critical mixtures. For all correlation lengths smaller than $\xi_{\mathrm{Gi}}$, the mixture shows mean field behaviour, for all correlation lengths larger than $\xi_{\mathrm{Gi}}$, fluctuations dominate and Ising behaviour can be observed. This is schematically visualised in Scheme 1. On the coexistence curve the relations $\nu=\nu_{-}$and $\xi_{0}^{-} / \xi_{0}=$ $0.51^{21}$ lead to a Ginzburg number $\mathrm{Gi}_{\text {coex }}=0.51 \mathrm{Gi}$ (see also Scheme 1).

\section{Experimental section}

\section{A. Samples}

The samples were commercial products obtained from Merck (Darmstadt) and Aldrich Chemie (Stuttgart). Their molar

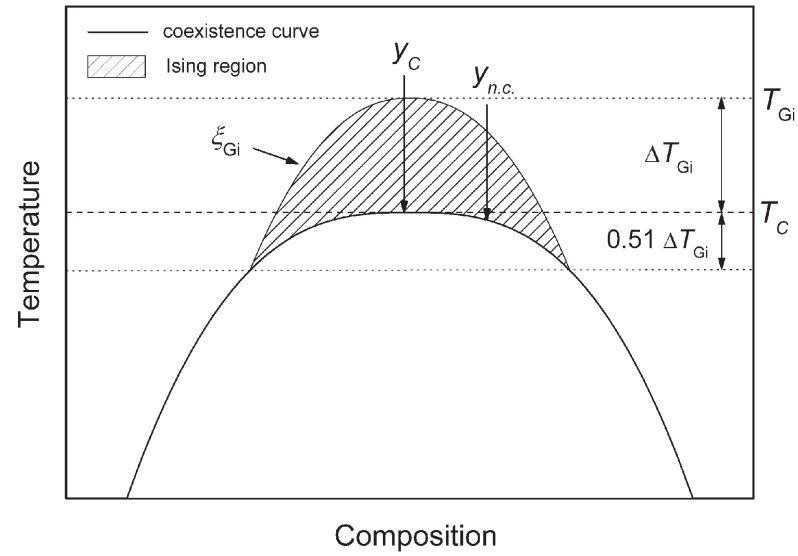

Scheme 1

mass distribution was characterised by MALDI (matrix assisted laser desorption ionisation) mass spectroscopy (for polyethylene glycol: $M_{N}=577 \mathrm{~g} \mathrm{~mol}^{-1}, M_{\mathrm{w}}=598 \mathrm{~g} \mathrm{~mol}^{-1}$, $M_{\mathrm{w}} / M_{N}=1.036$, for polypropylene glycol: $M_{N}=994 \mathrm{~g}$ $\left.\mathrm{mol}^{-1}, M_{\mathrm{w}}=1019 \mathrm{~g} \mathrm{~mol}^{-1}, M_{\mathrm{w}} / M_{N}=1.025\right) .{ }^{34}$ The cloud point curve was measured with a small angle light scattering setup described before. ${ }^{34}$ The composition of the critical mixture, $y_{\mathrm{PPG}, \mathrm{c}}=0.46$ ( $y_{\mathrm{PPG}}$ : mass fraction of polypropylene glycol), was determined by the criterion of equal volumes in the vicinity of its phase separation temperature. Additionally, the coexistence curves for the two investigated mixtures, the critical mixture and the non-critical mixture (mass concentration of PPG: $y_{\mathrm{PPG}}=0.365$ ), were determined by differential refractometry. The measurements of the coexistence curves are described in ref. 19.

\section{B. Light scattering experiments}

Proper amounts of the polymers were filtered through Millipore filters $(0.2 \mu \mathrm{m})$ into a dust free light scattering cell. The mixtures were prepared by weight. The homogenised sample was put into the light scattering set-up and held on constant temperature until no further change in the scattered light intensity could be observed. For measurements in the twophase region below $T_{\mathrm{c}}$, macroscopic phase separation had to be reached, resulting in a typical waiting time of one week prior to each measurement. The two coexisting phases - an upper, PPG-rich phase and a lower, PEG-rich phase in the light scattering cell-were measured one after another by shifting the cell so that the laser beam went through the respective measured phase. The light scattering experiments were performed with a commercial photometer (ALV, Langen). The light source was a $\mathrm{He}-\mathrm{Ne}$ laser (Spectra Physics, model 127) operating at $632.8 \mathrm{~nm}$ with a power of $35 \mathrm{~mW}$. The sample was measured in an angular range of $30^{\circ} \leq \theta \leq 150^{\circ}$, with $\theta$ being the scattering angle. This corresponds to a scattering vector range of $7.45 \times 10^{-3} \mathrm{~nm}^{-1} \leq q \leq 2.78 \times 10^{-2} \mathrm{~nm}^{-1}$ $(q=(4 \pi n / \lambda) \sin (\theta / 2))$. The temperature was controlled within an accuracy of $\pm 0.02 \mathrm{~K}$. The incident and the scattered laser beam were polarised perpendicular to the scattering plane (VV geometry). The dynamic light scattering experiments were performed with a correlator (ALV, model 5000/E) in the same angular range as the static experiments.

In a quasielastic light scattering experiment, the desired autocorrelation function of the scattered field $g_{1}(\boldsymbol{q}, t)$ is related to the measured intensity time autocorrelation function $G_{2}(\boldsymbol{q}, t)$ through the Siegert relation for the homodyne case:

$$
g_{2}(\boldsymbol{q}, t) \equiv G_{2}(\boldsymbol{q}, t) /\langle I(\boldsymbol{q}, t)\rangle^{2}=1+f \alpha^{2}\left|g_{1}(\boldsymbol{q}, t)\right|^{2},
$$

where $\langle I(\boldsymbol{q}, t)\rangle$ is the mean intensity, $f$ is an instrumental factor, and $\alpha$ is the fraction of the totally scattered intensity arising 
from concentration fluctuations $\left(\alpha=I_{\mathrm{cf}}(\boldsymbol{q}) / I(\boldsymbol{q})\right)$. In this case, the field correlation function $g_{1}(\boldsymbol{q}, t)$ is identified as the concentration autocorrelation function $\left(g_{1}(\boldsymbol{q}, t)=\langle\delta \phi(\boldsymbol{q}, t) \delta \phi(-\boldsymbol{q}, t)\rangle /\right.$ $\left\langle|\delta \phi(\boldsymbol{q}, 0)|^{2}\right\rangle,\left\langle|\delta \phi(\boldsymbol{q}, 0)|^{2}\right\rangle$ : mean square concentration fluctuations). $g_{1}(\boldsymbol{q}, t)$ is further given by ${ }^{35}$

$$
g_{1}(\boldsymbol{q}, t)=\frac{S(\boldsymbol{q}, t)}{S(\boldsymbol{q})},
$$

with the dynamic structure factor $S(\boldsymbol{q}, t)$ and the static structure factor $S(\boldsymbol{q})=\langle\delta \phi(\boldsymbol{q}, 0) \delta \phi(-\boldsymbol{q}, 0)\rangle$. The decay rate $\Gamma(\boldsymbol{q})$ of $S(\boldsymbol{q}, t)$ as defined in eqn. (7) is identical to the Rayleigh linewidth to be identified with the collective thermal decay rate of composition fluctuations $\delta \phi(\boldsymbol{q}, t)$. In the absence of mode coupling effects, the measured $\Gamma(\boldsymbol{q})=D q^{2}$ is related to the mutual diffusion coefficient $D$ as given by eqn. (7).

\section{Results and discussion}

\section{A. Phase diagram}

In Fig. 1, the cloud point curve of the system PEG600/ PPG1000 is shown. The measurements are described in ref. 34. Together with the cloud point curve (triangles), the coexistence curves of the two investigated mixtures, the critical mixture $\left(y_{\mathrm{PPG}}=0.46\right.$, solid circles $)$ and the non-critical mixture $\left(y_{\mathrm{PPG}}=0.365\right.$, open circles) are plotted. The coexistence curves were measured by differential refractometry (see ref. 19 where also the coexistence curves of three additional compositions are shown). The results for the phase diagram are interpreted by the influence of clusters built by intermolecular hydrogen bonds which lead to an effective polydispersity of the mixture. The analysis of the data is described in detail in ref. 19.

To the measured coexistence curves of the critical and the non-critical mixtures, the equation for the order parameter $\left(\left|y_{\mathrm{I}}-y_{\mathrm{II}}\right|=B_{0}(-\varepsilon)^{\beta}\right)$ was fitted, with the mass concentrations of the demixed phases $y_{\mathrm{I}}$ and $y_{\mathrm{II}}$, the reduced temperature $\varepsilon=\left(T-T_{\mathrm{c}}\right) / T_{\mathrm{c}}$ (where $T_{\mathrm{c}}$ is also a free fit parameter), the critical exponent of the order parameter $\beta$, and the critical amplitude $B_{0}$. The fitting curves are shown in Fig. 1 together with the measured coexistence curves. The results for the parameters $\beta$ and $B_{0}$ were $\beta=0.357 \pm 0.08$ and $B_{0}=0.81 \pm 0.03$ for the critical mixture and $\beta=0.497 \pm 0.03$ and $B_{0}=$ $1.16 \pm 0.1$ for the non-critical mixture. The critical exponent for the coexistence curve of the critical mixture is close to the Ising value of $\beta=0.325 .^{36}$ The critical exponent of the

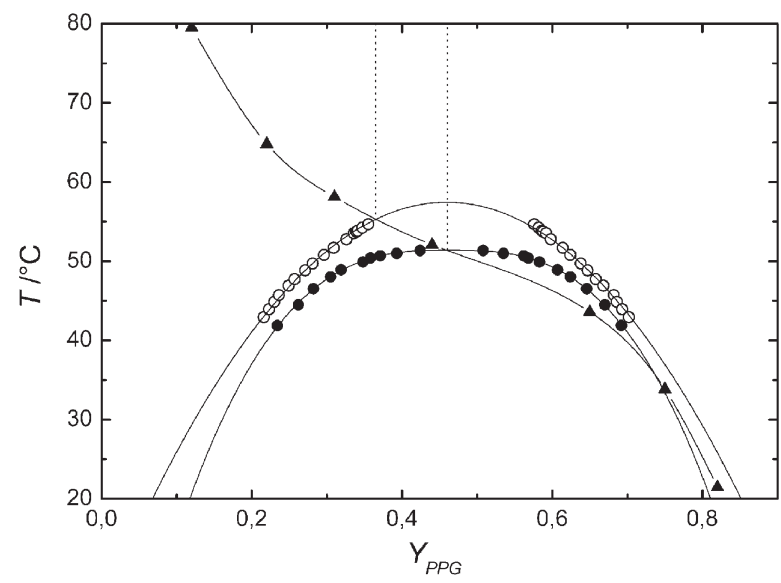

Fig. 1 Cloud point curve and phase coexistence curves of the mixture PEG600/PPG1000. Triangles: cloud point curve, ${ }^{34}$ solid circles: phase coexistence curve of the mixture with $y_{\mathrm{PPG}}=0.46$ (critical mixture), open circles: phase coexistence curve of the mixture with $y_{\mathrm{PPG}}=0.365$. The dotted lines indicate the compositions of the mixtures in the one-phase region. non-critical mixture corresponds with the mean field value $\beta=0.5$ within the experimental error limits.

\section{B. Light scattering on the critical mixture}

1. Correlation length. The results of the static light scattering measurements of the critical mixture in the homogeneous state and in the two-phase region are shown in Fig. 2. The reciprocal value of the scattered intensity is plotted as a function of the square of the scattering vector (Ornstein-Zernike plot). The data are well represented by straight lines. A fit of eqn. (4) to the data yields the correlation length $\xi$ of the composition fluctuations.

In Fig. 3, the correlation length obtained from the OrnsteinZernike plots is represented as a function of temperature. The value of the critical temperature $T_{\mathrm{c}}$ was obtained by fitting the power law $\xi=\xi_{0} \varepsilon^{-\nu}$ (eqn. (5)) to the data in the one-phase region and setting the theoretical value $\nu=0.63$ (3d-Ising value) as a fixed value. The fit yielded a critical temperature of $T_{\mathrm{c}}=(51.285 \pm 0.003)^{\circ} \mathrm{C}$ and a critical amplitude of $\xi_{0}=$ $(0.53 \pm 0.01) \mathrm{nm}$. The fit with the Ising value for the critical exponent $\nu$ describes the data in the one-phase region very well. The value for $\xi_{0}$ fits nicely to the already existing data for $\xi_{0}=f(N)^{37}$ if we use $\bar{N}=\sqrt{ }\left(N_{1} N_{2}\right)=15.4$. This is close to the number of statistical segments in the PEG and PPG oligomers (see below).

In the two-phase region, the equation $\xi=\xi_{0}^{-}|\varepsilon|^{-\nu_{-}}$(eqn. (6)) was fitted to the data, also with $\nu=0.63$ as a fixed value in order to test the theoretical amplitude ratio of $\xi_{0}^{-} / \xi_{0}=$ 0.51 predicted by the Ising model. ${ }^{21}$ The results were $\xi_{0}^{-}=$ $(0.27 \pm 0.003) \mathrm{nm}$ in the PPG-rich phase and $\xi_{0}^{-}=(0.29 \pm$ $0.03) \mathrm{nm}$ in the PEG-rich phase, leading to $\xi_{0}^{-} / \xi_{0}=0.51 \pm$ 0.02 for the PPG-rich phase and $\xi_{0}^{-} / \xi_{0}=0.55 \pm 0.07$ for the
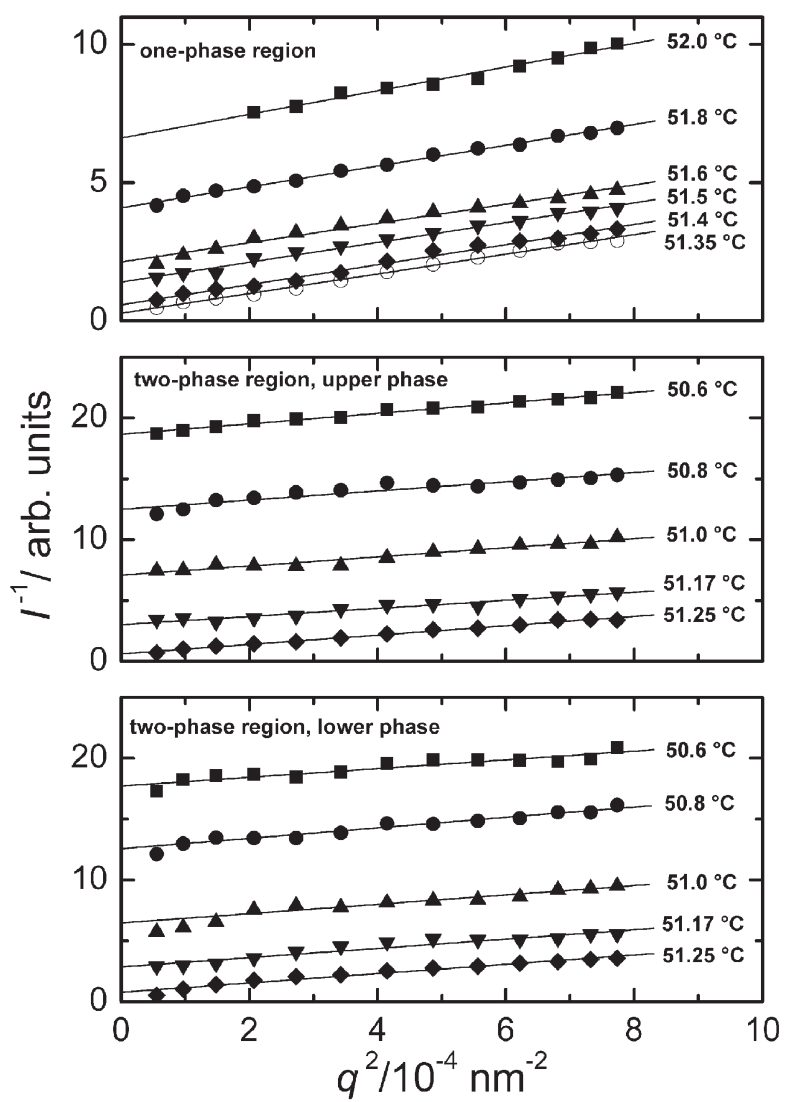

Fig. 2 Ornstein-Zernike plot of the scattered light intensity of the critical mixture for various temperatures in the one-phase region and in the coexisting phases (upper phase: PPG-rich phase, lower phase: PEG-rich phase). 


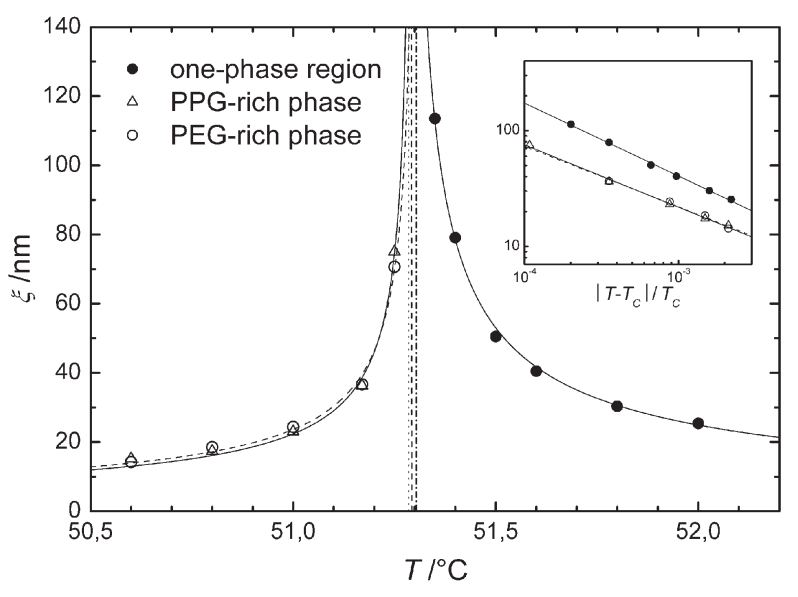

Fig. 3 Correlation length $\xi$ of the concentration fluctuations of the critical mixture as a function of temperature. The curves through the data points represent the related fitting functions (eqn. (5) for the one-phase region and eqn. (6) for the two-phase region) with the Ising value of $\nu=0.63$ as a fixed parameter (dashed curve: data of the PEG-rich phase). The straight vertical lines denote the critical temperature $T_{\mathrm{c}}$ from the fitting procedures (dotted line: one-phase region, dashed line: PPG-rich phase, dashed-dotted line: PEG-rich phase). The inset shows the data in a $\log \xi$ vs. $\log \varepsilon$ plot.

PEG-rich phase. The critical temperatures from the fits in the two-phase region were $T_{\mathrm{c}}=(51.29 \pm 0.03)^{\circ} \mathrm{C}$ in the PPG-rich phase and $T_{\mathrm{c}}=(51.30 \pm 0.04)^{\circ} \mathrm{C}$ in the PEG-rich phase, which agree with the value from the fit in the one-phase region within the experimental error limits. The straight dashed, dotted, and dashed-dotted lines in Fig. 3 depict the values for the critical temperature from the fits.

In order to test whether the data are described better by the mean field predictions or by the Ising model, fits with the mean field value $\nu=0.5$ to the data in the one- and two phase region have been also made (not shown). The corresponding fit values were $T_{\mathrm{c}}=(51.32 \pm 0.01)^{\circ} \mathrm{C}$ and $\xi_{0}^{-}=(1.15 \pm 0.04) \mathrm{nm}$ in the one-phase region, $T_{\mathrm{c}}=(51.28 \pm 0.01)^{\circ} \mathrm{C}$ and $\xi_{0}^{-}=(0.67 \pm$ $0.02) \mathrm{nm}$ in the PPG-rich phase, and $T_{\mathrm{c}}=(51.28 \pm 0.02)^{\circ} \mathrm{C}$ and $\xi_{0}^{-}=(0.70 \pm 0.03) \mathrm{nm}$ in the PEG-rich phase. The fitting curves with the mean field value $\nu=0.5$ described the data rather well but the results for the amplitude ratios $\xi_{0}^{-} / \xi_{0}$ from these fits $\left(\xi_{0}^{-} / \xi_{0}=0.58 \pm 0.04\right.$ for the PPG-rich phase and $\xi_{0}^{-} / \xi_{0}=0.61 \pm 0.05$ for the PEG-rich phase) differed from the value of the mean field theory $\left(\xi_{0}^{-} / \xi_{0}=1 / \sqrt{ } 2 \approx 0.71\right)$.
Moreover the values for $T_{\mathrm{c}}$ from the mean field fits did not coincide for the data in the one- and two-phase region. This allows for the conclusion that the data are described better by the Ising model. The theoretical prediction of the Ising model for the critical amplitude ratio $\xi_{0}^{-} / \xi$ could be confirmed experimentally for a polymer/oligomer mixture for the first time to our knowledge.

In a measurement of the correlation length of a polymer mixture by neutron scattering ${ }^{11}$ where the upper and the lower phase could not be measured separately, the measured amplitude ratios differed from the theoretical Ising value by $20 \%$ which was outside the experimental error limit. Since those measurements averaged over the fluctuations in both phases of the two-phase region and the assumption of a thermodynamic equilibrium for the coexisting phases is not justified, the results should be considered with some care.

2. Mutual diffusion coefficient. The diffusion coefficient in the hydrodynamic region $(q \xi<1)$ is obtained from the measured Rayleigh linewidth with eqns. (8) and (9) as shown in 1:

$$
D \cong D_{\mathrm{c}}=\frac{\Gamma(\boldsymbol{q})}{q^{2}} \frac{1}{K(x)}
$$

However, eqn. (16) is also valid for $q \xi \geq 1$, the mode coupling region close to $T_{\mathrm{c}}$. We have performed the measurements in the region close to $T_{\mathrm{c}}$ and Fig. 4 shows the respective diffusion coefficients for different temperatures in accordance to eqn. (16) in an $\varepsilon$ range of $10^{-4}<\varepsilon<10^{-2}(0.095 \leq q \xi \leq 3.15)$. According to the mode coupling correction $K(x)$, all data for different temperatures fall on straight lines versus $q^{2}$ which allows to establish $D_{\mathrm{c}}(q)=f(T)$.

In Fig. 5, the diffusion coefficient $D$ in the one- and twophase region is shown as a function of temperature. The power law $D=D_{0} \varepsilon^{\nu^{*}}$ (eqn. (10)) was fitted to the data in the onephase region by setting $T_{\mathrm{c}}=51.285^{\circ} \mathrm{C}$, the value obtained by static light scattering, as a fixed value. The result of the fitting procedure for the critical exponent is $\nu^{*}=0.70 \pm 0.03$, which coincides with the theoretical value $\nu^{*}=0.67$ for the $3 \mathrm{~d}$-Ising model within the experimental error limits. The result for the critical amplitude is $D_{0}=(1.8 \pm 0.3) \times 10^{-7} \mathrm{~cm}^{2} \mathrm{~s}^{-1}$.

The equation $D=D_{0}^{-}|\varepsilon|^{\nu_{-}^{*}}$ (eqn. (11)) was fitted to the data in the two-phase region by setting $T_{\mathrm{c}}=51.285^{\circ} \mathrm{C}$ as a fixed value. The results for the critical exponents were $\nu_{-}^{*}=$ $0.57 \pm 0.02$ for the PPG-rich phase and $\nu_{-}^{*}=0.56 \pm$ 0.02 for the PEG-rich phase. The critical amplitudes are

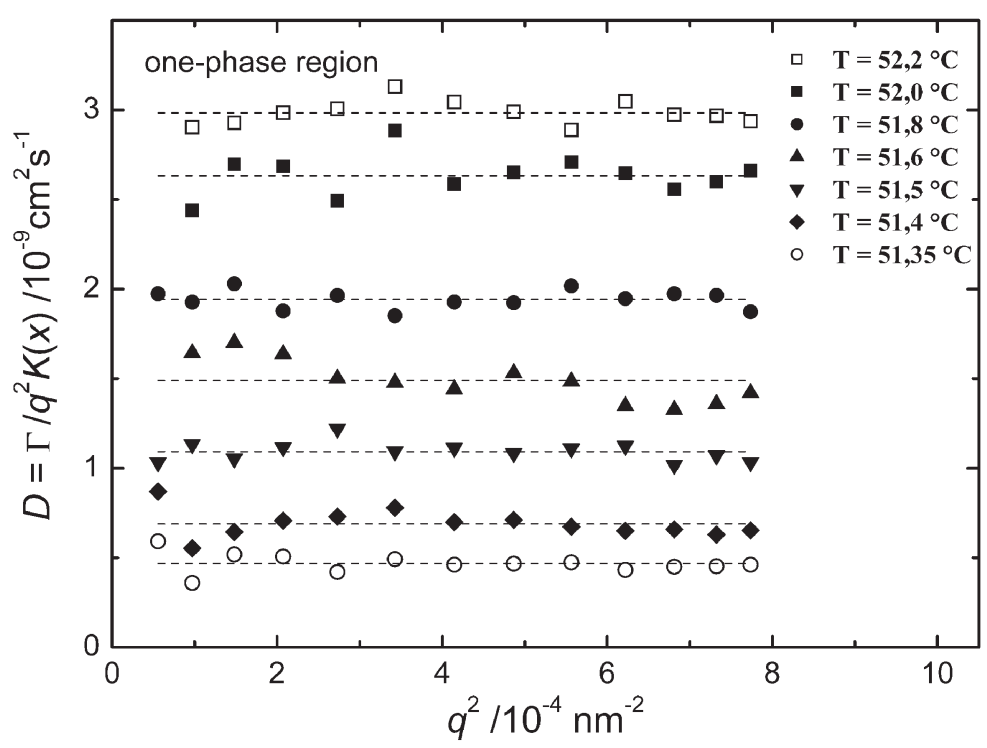

Fig. 4 Mutual diffusion coefficient $D$ calculated from eqn. (16) for different temperatures in the one phase region as a function of $q^{2}$. 


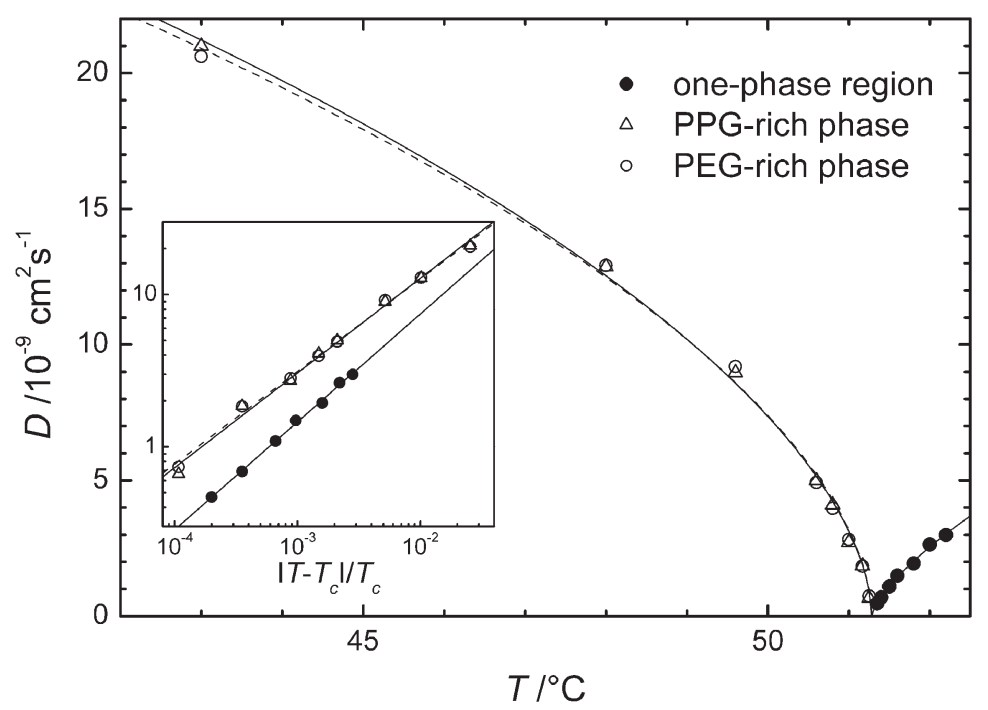

Fig. 5 Mutual diffusion coefficient $D$ of the critical mixture as a function of the reduced temperature $\varepsilon$. The curves represent the fitting functions (eqn. (10) for the one-phase region, eqn. (11) for the two-phase region, dashed curve: PPG-rich phase). The results of the fitting procedures are: $\nu^{*}=0.70, D_{0}=1.8 \times 10^{-7} \mathrm{~cm}^{2} \mathrm{~s}^{-1}$ in the one-phase region, $\nu_{-}^{*}=0.57, D_{0}=1.7 \times 10^{-7} \mathrm{~cm}^{2} \mathrm{~s}^{-1}$ in the PPG-rich phase, and $\nu_{-}^{*}=0.56$, $D_{0}=1.6 \times 10^{-7} \mathrm{~cm}^{2} \mathrm{~s}^{-1}$ in the PEG-rich phase. In the inset, a log-log plot of the data in the one- and two-phase regions and the corresponding fits are shown as a function of the absolute value of the reduced temperature $\varepsilon$.

approximately the same in the homogeneous and decomposed state $\left(D_{0}=(1.8 \pm 0.3) \times 10^{-7} \mathrm{~cm}^{2} \mathrm{~s}^{-1}\right.$ in the one-phase region, $D_{0}^{-}=(1.7 \pm 0.1) \times 10^{-7} \mathrm{~cm}^{2}$ in the PPG-rich phase, $D_{0}^{-}=$ $(1.6 \pm 0.1) \cdot 10^{-7} \mathrm{~cm}^{2} \mathrm{~s}^{-1}$ in the PEG-rich phase). In the inset of Fig. 5, a log-log plot of the diffusion coefficient in the one- and two-phase region and the corresponding fits are shown as a function of the absolute value of the reduced temperature $\varepsilon$. Fits with the Ising value $\nu^{*}=0.67$ (and $T_{\mathrm{c}}$ as a free parameter) could not describe the data in the two-phase region in the whole temperature range. So the critical exponents show a tendency to mean field behaviour in the two-phase region. The results of the fits for the critical mixture are summarised in Table 1.

\section{Light scattering on the non-critical mixture}

The non-critical mixture with the concentration $y_{\mathrm{PPG}}=0.365$ was measured by light scattering in a temperature region of $43.0^{\circ} \mathrm{C} \leq T \leq 54.8^{\circ} \mathrm{C}$. The temperature of demixing $T_{\mathrm{P}}$ was determined as $T_{\mathrm{P}}=52.80^{\circ} \mathrm{C}$.

1. Diffusion coefficient. In Fig. 6, the mutual diffusion coefficient in the one-phase region and in the two coexisting phases

Table 1 Critical exponents and amplitudes of the correlation length and the diffusion coefficient for the critical $\left(y_{\mathrm{PPG}}=0.46\right)$ and noncritical $\left(y_{\mathrm{PPG}}=0.365\right)$ mixture. For the values in parenthesis fixed parameters for the critical exponents were taken for the fits (see text)

\begin{tabular}{|c|c|c|c|c|c|}
\hline & & $\nu$ & $\xi_{0} / \mathrm{nm}$ & $\nu^{*}$ & $\begin{array}{l}10^{7} D_{0} / \\
\mathrm{cm}^{2} \mathrm{~s}^{-1}\end{array}$ \\
\hline \multirow[t]{3}{*}{$\begin{array}{l}\text { Critical } \\
\text { mixture }\end{array}$} & $\begin{array}{l}\text { One phase } \\
\text { region }\end{array}$ & 0.63 & 0.53 & 0.70 & 1.8 \\
\hline & $\begin{array}{c}\text { PPG-rich } \\
\text { phase }\end{array}$ & 0.56 & 0.43 & 0.57 & 1.7 \\
\hline & $\begin{array}{c}\text { PEG-rich } \\
\text { phase }\end{array}$ & 0.52 & 0.59 & 0.56 & 1.6 \\
\hline \multirow[t]{3}{*}{$\begin{array}{l}\text { Non-critical } \\
\text { mixture }\end{array}$} & $\begin{array}{l}\text { One phase } \\
\text { region }\end{array}$ & $(0.50)$ & $(0.48 / 0.55)$ & 0.50 & 1.2 \\
\hline & $\begin{array}{l}\text { PPG-rich } \\
\text { phase }\end{array}$ & - & - & $(0.60)$ & (2.0) \\
\hline & $\begin{array}{l}\text { PEG-rich } \\
\text { phase }\end{array}$ & $(0.50)$ & $(0.83)$ & 0.53 & 1.5 \\
\hline
\end{tabular}

is shown as a function of temperature, together with the corresponding fit functions. The corresponding $\log D v s . \log \varepsilon$ plots to determine the scaling parameters are shown in Fig. 7. The measured diffusion coefficients of the upper and lower phase of the coexisting phases are usually plotted versus $\varepsilon=\left(T-T_{\mathrm{c}}\right.$, where $T_{\mathrm{c}}$ is used as the reference temperature. As shown in the phase diagram (Fig. 1) and discussed in detail in ref. 19, due to the broad "effective molar mass distribution" of the hydrogen bond clusters the reference temperatures are different due to the different coexistence curve related to the non-critical composition. For the analysis of the non-critical mixture by eqn. (10), we therefore replaced the reference temperature $T_{\mathrm{c}}$ by $T_{\mathrm{c}}$ which was used as a free fit parameter for the fit of the PEG-rich phase. The result of the three-parameter fit was a value of $T_{\mathrm{c}}=53.45^{\circ} \mathrm{C}$.

Since the volume of the PPG-rich phase (the minority phase) was very small, the diffusion coefficient could only be measured for $T_{\mathrm{P}}-T>2 \mathrm{~K}$. A three-parameter fit was not possible for the PPG-rich phase, so the value $T_{\mathrm{c}}^{\prime}=53.45^{\circ} \mathrm{C}$ from the fit of the PEG-rich phase was taken as a fixed parameter. The critical amplitudes and exponents determined by the fits in the two-phase region are summarised in Table 1.

The data in the one-phase region are analysed in the frame of the pseudospinodal concept. Thus the diffusion coefficient for the one-phase region is fitted in analogy to eqn. (12) by

$$
D=\tilde{D}_{0}\left(\frac{T-T_{\mathrm{PS}}}{T_{\mathrm{PS}}}\right)^{\tilde{\nu}^{*}} \text {. }
$$

The pseudospinodal temperature $\left(T_{\mathrm{PS}}=52.22^{\circ} \mathrm{C}\right)$ from the fit is indicated in Fig. 6 and the values for $\tilde{\nu}^{*}$ and $\tilde{D}$ are also given in Table 1.

As mentioned above, the diffusion coefficient of the PPGrich phase could only be measured for $T_{\mathrm{P}}-T>2 \mathrm{~K}$. Therefore the results from this fit are not very reliable. However, the fits for the diffusion coefficient for the one- and the twophase region of the non-critical mixture as shown in Table 1 show a clear tendency to mean field behaviour.

2. Correlation length. Fig. 8 shows the Ornstein-Zernike plots for the non-critical mixture in the one-phase region (upper part of Fig. 8) and in the lower, PEG-rich phase (lower part of Fig. 8). In the one-phase region, two processes could be detected by static light scattering, one arising from 


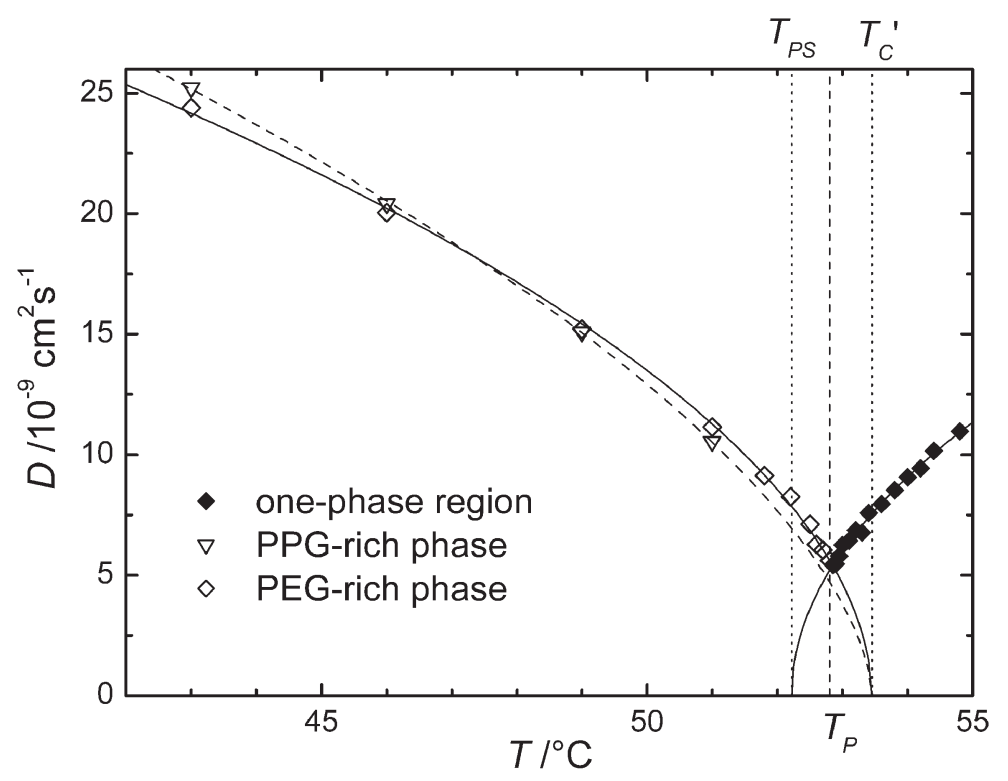

Fig. 6 Mutual diffusion coefficient $D$ of the non-critical mixture in the one-phase region and in both coexisting phases as a function of temperature. The curves represent the fitting functions to the data (dashed curve: PPG-rich phase). $T_{\mathrm{P}}$ is the temperature of demixing, $T_{\mathrm{PS}}$ the pseudospinodal temperature and $T_{\mathrm{c}}$ the virtual critical temperature of the coexistence curve belonging to the concentration $y_{\mathrm{PPG}}=0.365$ (for an explanation see text). The results of the fitting procedures in the two-phase region are:, $T_{\mathrm{c}}^{\prime}=53.45^{\circ} \mathrm{C}, \nu_{-}^{*}=0.53, D_{0}=1.5 \times 10^{-7} \mathrm{~cm}^{2} \mathrm{~s}^{-1}$ in the PEG-rich phase and $\nu_{-}^{*}=0.60, D_{0}=2.0 \times 10^{-7} \mathrm{~cm}^{2} \mathrm{~s}^{-1}$ in the PPG-rich phase.

concentration fluctuations, the other from clusters. ${ }^{19}$ The data shown in the upper part of Fig. 8 are only the contribution from concentration fluctuations. This fluctuation contribution to the total scattered intensity $I_{\mathrm{fl}}$ was calculated by subtraction of the scattering contribution of the clusters $I_{\text {cluster }}$ from the total scattered intensity $I$. The details of the estimation of $I_{\text {cluster }}$ are described in ref. 19.

For the non-critical mixture, the PPG-rich phase is the minority phase and could not be measured close to the temperature of demixing because the volume of the phase was too small. Measurements of the PPG-rich phase were only possible in the temperature range $43.0^{\circ} \mathrm{C} \leq T \leq 51.0^{\circ} \mathrm{C}$ where, due to the low scattering intensity only dynamic light scattering measurements could be carried out (see above).

Another possibility to determine the correlation length is to calculate it from dynamic light scattering and measured viscosity data, using the Stokes-Einstein relation, eqn. (9):

$$
\xi=R \frac{k_{\mathrm{B}} T}{6 \pi \eta D_{\mathrm{c}}} .
$$
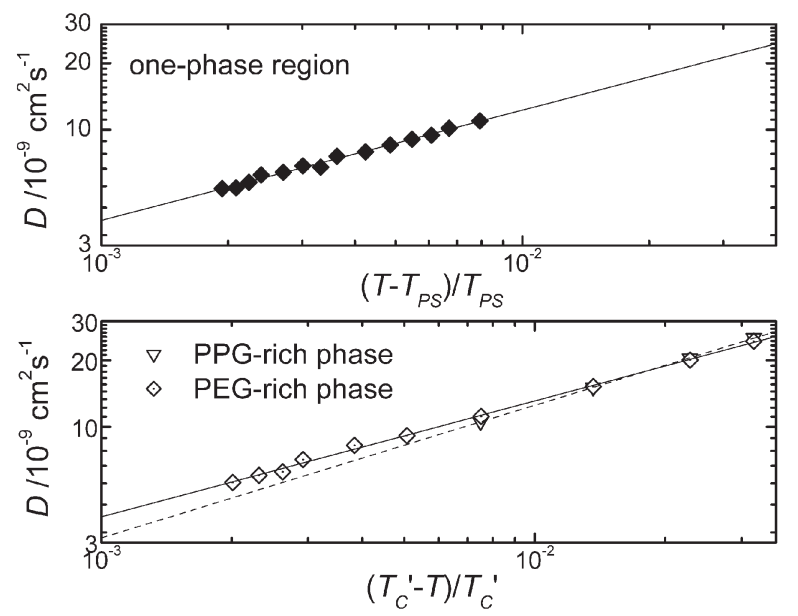

Fig. 7 Plot of $\log D$ vs. $\log \varepsilon$ of the non-critical mixture in the onephase (upper part) and the two-phase (lower part) region. The data are taken from Fig. 6.
In Fig. 9, the correlation length in the one-phase region and in the PEG-rich phase of the two coexisting phases is shown as a function of temperature, together with the related fit functions. The corresponding $\log \xi$ versus $\log \varepsilon$ plots are shown in Fig. 10.
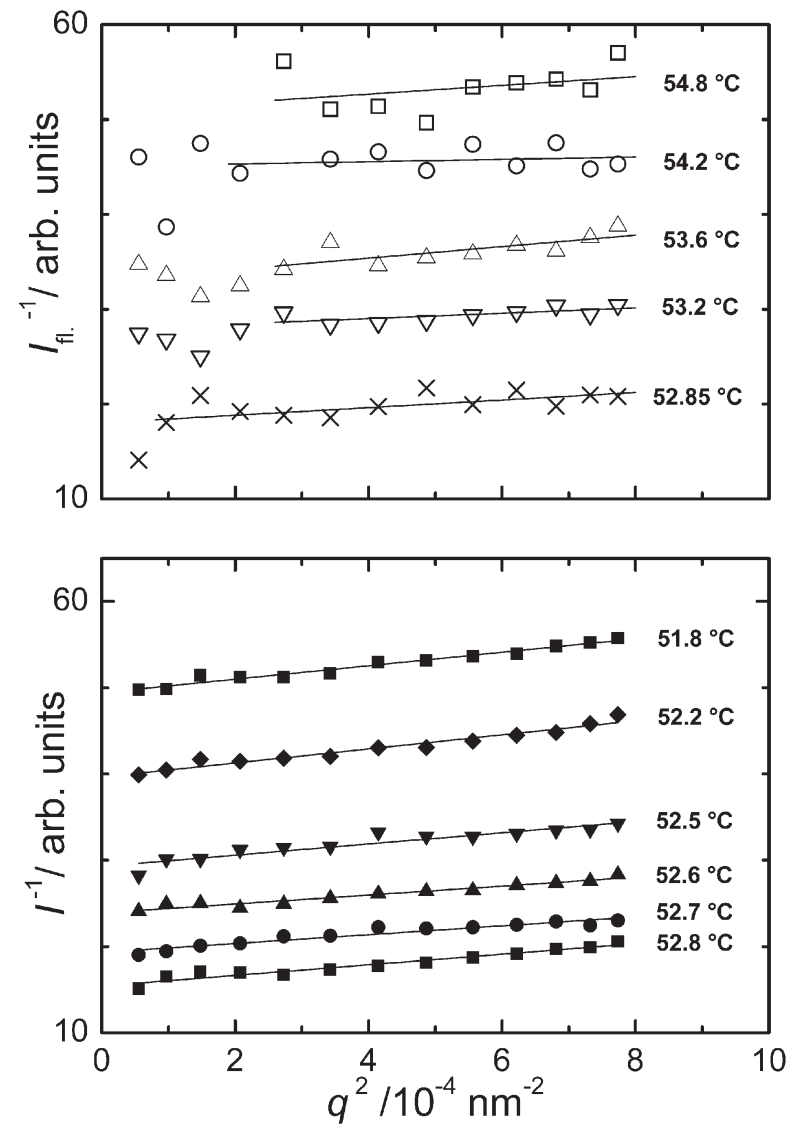

Fig. 8 Ornstein-Zernike plot of the scattered light intensity of the non-critical mixture in the one-phase region (upper part) and in the PEG-rich phase for selected temperatures (lower part). In the onephase region, the scattered intensity is corrected by subtracting the contribution of the clusters (see ref. 19). 


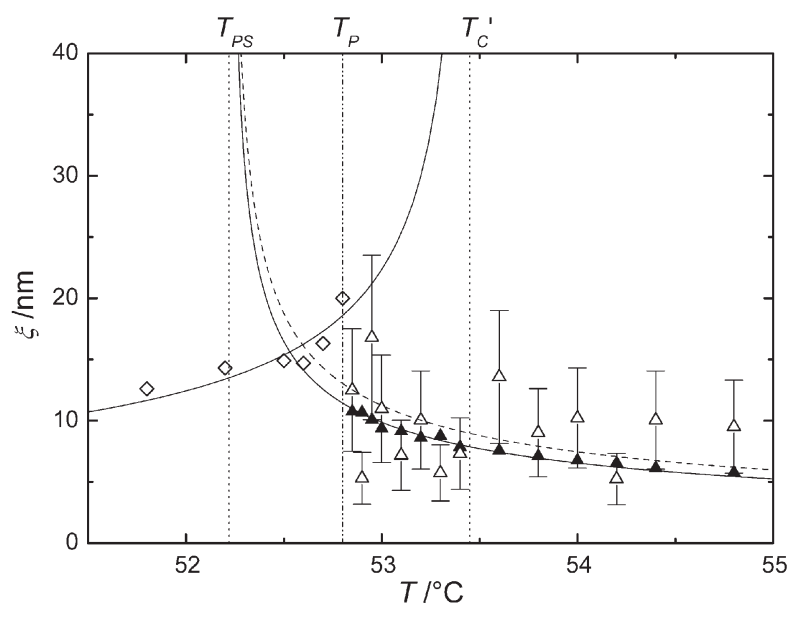

Fig. 9 Correlation length $\xi$ of the concentration fluctuations as a function of temperature for the non-critical composition in the onephase region $(\triangle, \boldsymbol{\Delta})$ and in the PEG-rich phase of the two-phase region $(\diamond)$. The open triangles refer to the Ornstein-Zernike analysis whereas the filled triangles refer to the Stokes-Einstein analysis. The values of the pseudospinodal temperature and the "critical" temperature $\left(T_{\mathrm{PS}}=52.22^{\circ} \mathrm{C}, T_{\mathrm{c}}=53.45^{\circ} \mathrm{C}\right)$ and the critical exponents $\left(\nu=\nu_{-}=\right.$ $0.50)$ are determined by dynamic light scattering. The fits to the data points of the non-critical composition with these values (solid lines) yield $\xi_{0}=0.48 \mathrm{~nm}$ in the one-phase region and $\xi_{0}^{-}=0.84 \mathrm{~nm}$ in the PEG-rich phase as results for the critical amplitudes.

The data in the one-phase region are analysed in the frame of the pseudospinodal concept. For the one-phase region, the data determined from the Ornstein-Zernike plot and those from the Stokes-Einstein analysis are shown together. As expected $^{38}$ the $\xi$-values for both types of analysis coincide within the error limits. Due to their higher accuracy only the dynamic light scattering data were taken for further analysis.

For the correlation length in the PEG-rich phase shown in Fig. 9 and in the lower part of Fig. 10 (open diamonds), a description with a power law $\xi=\xi_{0}^{-}|\varepsilon|^{-\nu_{-}}$(eqn. (6) with $\left.\varepsilon=\left(T-T_{\mathrm{c}}^{\prime}\right) / T_{\mathrm{c}}^{\prime}\right)$ was possible (solid line). Because of the small number of data points and the relatively weak dependence of the correlation length on temperature, a three-parameter fit was not possible, therefore the "critical temperature" $T_{\mathrm{c}}^{\prime}$ and the critical exponent had to be taken as fixed values. For $T_{\mathrm{c}}$, the result of the fitting of the diffusion coefficient $\left(T_{\mathrm{c}}^{\prime}=53.45^{\circ} \mathrm{C}\right)$ was used. For the critical exponent, the mean field value $\left(\nu_{-}=0.50\right)$ was taken because a tendency to mean
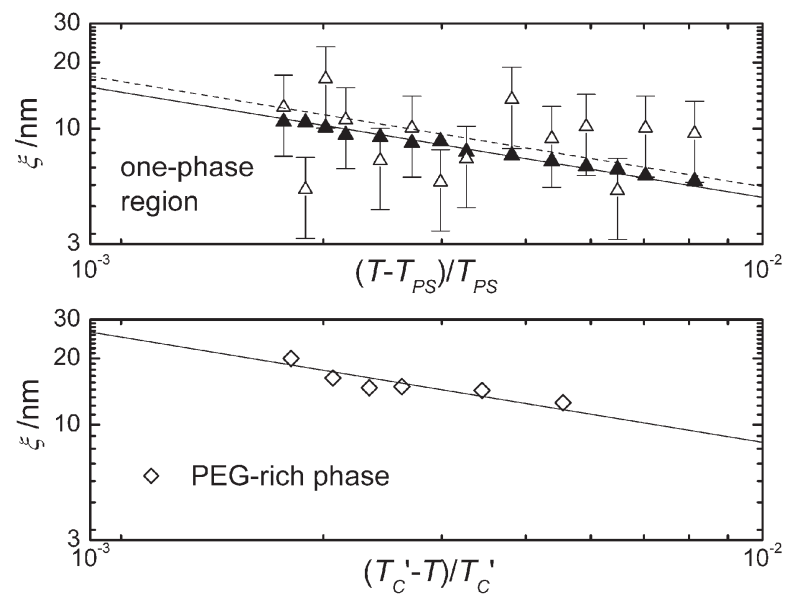

Fig. 10 Log-log plot of the correlation length of the non-critical mixture in the one-phase region (upper part) and two-phase region (lower part) as a function of the reduced temperature. The data are taken from Fig. 9. field behaviour was found for the diffusion coefficient. The result of a fit of eqn. (6) with these values to the data of the PEG-rich phase was a critical amplitude of $\xi_{0}^{-}=(0.83 \pm 0.03)$ $\pm 0.03) \mathrm{nm}$.

The correlation length for the one-phase region determined by dynamic light scattering and viscosity data (solid triangles) and by static light scattering and subtraction of the contribution of the clusters (open triangles) are shown in Fig. 9 and the upper part of Fig. 10 as a function of temperature. The plotted line represents the fit function $\xi=\xi_{0} \varepsilon^{-\nu}$, with $\varepsilon=\left(T-T_{\mathrm{PS}}\right) / T_{\mathrm{PS}}$ (eqn. (12)), with the values for the pseudospinodal temperature and the critical exponent $\left(T_{\mathrm{PS}}=\right.$ $52.22^{\circ} \mathrm{C}, \tilde{\nu}=0.50$ ) obtained by dynamic light scattering. From this fitting procedure, the values $\tilde{\xi}_{0}=(0.48 \pm 0.04) \mathrm{nm}$ (Stokes-Einstein analysis using dynamic light scattering and viscosity data) and $\tilde{\xi}_{0}=(0.55 \pm 0.05) \mathrm{nm}$ (Ornstein-Zernike analysis: static light scattering data after subtraction of the cluster contribution) were determined for the critical amplitude. The critical exponents and amplitudes for the correlation length are also summarised in Table 1.

\section{Critical fluctuations and hydrogen bond clusters}

1. Ginzburg number and hydrogen bond clusters. After an analysis of the static and dynamic light scattering data for both critical and non-critical mixture (in the one-phase region and in the coexisting phases of the two-phase region) the question arises how the clusters formed by hydrogen bonds influence the critical behaviour of the mixture. As discussed already in the introduction and in Section II C the Ginzburg number $\mathrm{Gi}$ is a measure for the reduced temperature $\varepsilon$ where the crossover from Ising $\left(\varepsilon=\left(T-T_{\mathrm{c}}\right) / T_{\mathrm{c}}<\mathrm{Gi}\right)$ to mean field behaviour $\left(\varepsilon=\left(T-T_{\mathrm{c}}\right) / T_{\mathrm{c}}>\mathrm{Gi}\right)$ occurs. For mixtures of samples with low molecular mass $\mathrm{Gi}$ is in the order of 0.01 to $0.04,{ }^{18}$ whereas for mixtures of high molecular mass polymers $\mathrm{Gi}$ is predicted to be inversely proportional to the number of statistical segments $\left(\mathrm{Gi} \cong 1 / N_{a}\right)$. This is schematically shown in Scheme 2 where the curve $\varepsilon\left(N_{a}\right)=\operatorname{Gi}\left(N_{a}\right)$ divides the $\varepsilon-N_{a}$ plane into an upper region, where the mean field theory describes the critical behaviour, and a lower region, where the Ising exponents are expected to fit to the data.

For a fixed reduced temperature distance to the critical temperature $(\varepsilon=$ constant $)$ an increase of the degree of polymerisation can therefore result in a crossover from Ising to mean field behaviour (see horizontal arrow in Scheme 2). Following this idea, one can expect that an increase of the "effective molar mass" due to the formation of intermolecular hydrogen bonds can lead to mean field exponents although $N_{a}$ of the

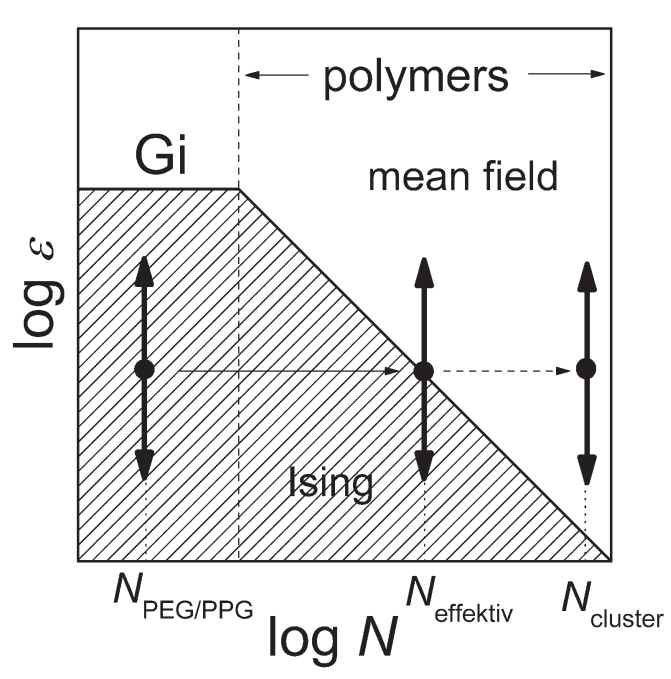

Scheme 2

Phys. Chem. Chem. Phys., 2003, 5, 3202-3211 
oligomers would suggest an Ising type of behaviour. In order to test this expectation we calculated the Ginzburg numbers for the PPG/PEG mixture using (i) the molar mass of the oligomers and (ii) the molar mass of the hydrogen bond clusters.

The number of statistical segments of PEG and PPG oligomers can be calculated by using the average molar mass measured by MALDI (see Sec. III A), the length of the statistical segments and the characteristic ratio $c_{\infty}$ given by Aharoni ${ }^{39}$ to be $N_{a, \text { PEG }}=25$ and $N_{a \text {,PPG }}=29$ for PEG and PPG, respectively. With $G i \cong 1 / N_{a}$, this yields a Ginzburg number $\mathrm{Gi}=$ 0.037 for a mixture of equal portions of PEG and PPG which leads to $T_{\mathrm{Gi}}-T_{\mathrm{c}} \approx 12 \mathrm{~K}$. An alternative calculation of the Ginzburg number is to use the relations $\xi_{\mathrm{Gi}}=R_{\mathrm{g}}\left(\bar{N}_{a}\right)^{1 / 2}$ and $\xi_{\mathrm{Gi}}=\xi_{0} \varepsilon_{\mathrm{Gi}}^{-\nu}\left(\xi_{0}\right.$ and $\nu$ are taken from the fit of eqn. (19)) which yields $\varepsilon_{\mathrm{Gi}}=\mathrm{Gi}=0.031$ and $T_{\mathrm{Gi}}-T \approx 10 \mathrm{~K} . R_{\mathrm{g}}$ and $\bar{N}_{a}$ are again calculated using the values from reference. ${ }^{39}$ Following the prediction of the renormalization group theory ${ }^{21}$ the Ginzburg number for the cexistence curve is $\mathrm{Gi}_{\text {coex }}=0.51 \mathrm{Gi}$ which yields $\left|T_{\mathrm{Gi} \text {,coex }}-T_{\mathrm{c}}\right| \cong 5.1 \mathrm{~K}$.

For the hydrogen bond clusters measured by light scattering ${ }^{19}$ the radius of gyration of the clusters $R_{\mathrm{g}, \text { cluster was found }}$ to be in the order of 250 to $550 \mathrm{~nm}$. Assuming a statistical coil for the form of the clusters, with $R_{\mathrm{g}, \text { cluster }}=a\left(N_{a \text {,cluster }} / 6\right)^{1 / 2}$ ( $R_{\mathrm{g}, \text { cluster }}$ : radius of gyration of the clusters, $a$ : length of a statistical segment, $N_{a}$ : number of the statistical segments) and $\mathrm{Gi} \cong 1 / N_{a}$,Cluster a value of $\mathrm{Gi} \approx 2 \times 10^{-7}$ can then be estimated by assuming $100 \%$ clusters in the mixture. This yields a temperature distance $T_{\mathrm{Gi}}-T_{\mathrm{c}}$ for the crossover which is much smaller than $0.01 \mathrm{mK}$. However, this estimation is not realistic since the clusters were only detected in the PPG-rich phase and not all oligomers are expected to belong to the clusters. ${ }^{40}$ From the shift of the critical molar mass for entanglements due to hydrogen bond clusters in PPG melts as discussed in ${ }^{40}$ one can conclude that the "effective molar mass" for hydrogen bond clusters formed by PPG oligomers is by a factor of about 5 larger than the molar mass of the oligomers which would yield to a Ginzburg number $\mathrm{Gi} \approx 0.01$ and $T_{\mathrm{Gi}}-T_{\mathrm{c}} \approx 3 \mathrm{~K}$. This would decrease the temperature region were the Ising model is valid from about $T_{G i}-T_{\mathrm{c}} \approx 10 \mathrm{~K}$ for assuming free oligomers to $T_{\mathrm{Gi}}-T_{\mathrm{c}}<3 \mathrm{~K}$ for the hydrogen bond clusters.

The situation for the assumption of hydrogen bond clusters is visualized schematically in the phase diagram in Fig. 11. The hatched area represents the "Ising region" in the one phase region by assuming hydrogen bond clusters. The corresponding "Ising region" of the coexistence curve for the critical

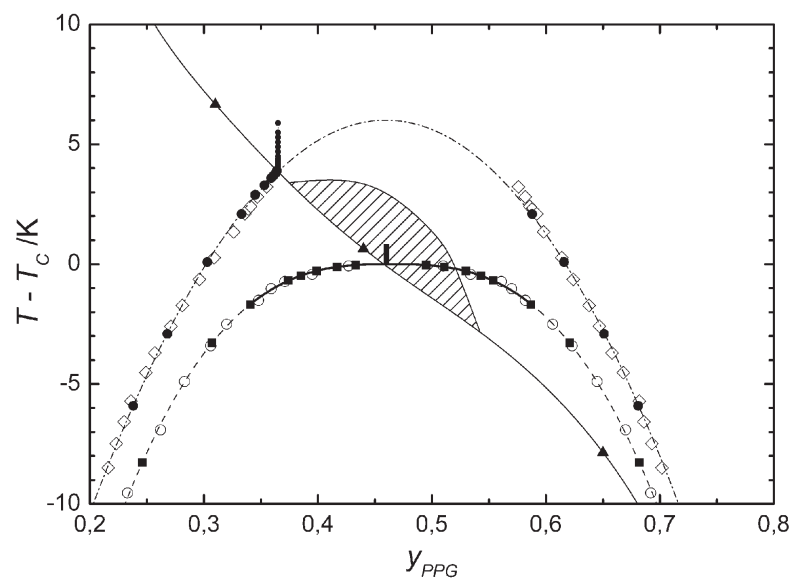

Fig. 11 Coexistence curves (open symbols taken from Fig. 1) for the critical and non-critical mixture are shown together with the compositions and temperatures were the light scattering experiments (solid symbols) have been performed. Further the schematic depiction of the Ising region in the one-phase region (hatched part of the figure) and in the two coexisting phases for the critical mixture (solid part of the coexistence curve) are shown. mixture is indicated by the solid line on the coexistence curve (region where $\left|T_{\mathrm{Gi} \text {,coex }}-T_{\mathrm{c}}\right|<0.51 \mathrm{Gi}$ ). The location of the hatched area is justified by two findings: (i) the critical mixture in the one-phase region exhibits only Ising behaviour whereas (ii) the data of the non-critical mixture in the one-phase region suggests mean field type of behaviour (Table 1).

Additional support for the tendency to mean-field type of behaviour comes from the analysis of the critical exponents in the two-phase region (see also Table 1). All values for $\nu$ and $\nu^{*}$ show a tendency towards the mean-field value of 0.5 and are undoubtedly different from the Ising values within the error limits. The critical exponents for the coexistence curves (see Section IV A) suggest a broad crossover region which is probably due to the large "effective molar mass distribution" of the clusters as predicted in 40 . From that we conclude that the Ising region suggested in Fig. 11 is rather an upper estimation.

\section{Conclusion}

In this study, we have performed light scattering experiments in a critical and a non-critical mixture of PPG and PEG oligomers. The dynamic and static light scattering experiments were performed in the one phase region as well as in the PPG-rich and PEG-rich phase of the two-phase region (coexisting phases). From the temperature dependence of the correlation length $\xi$ and the diffusion coefficient $D$ the critical amplitudes and exponents were determined. The main results from those experiments are the following:

(i) The formation of intermolecular hydrogen bond structures leads to an increase of the effective molar mass and its distribution, which is responsible for the phase behaviour of the "pseudo-binary mixture". These hydrogen bond clusters influence the critical behaviour in a similar way as known from polymers with high molar mass, i.e. the temperature and composition region near the critical point where the critical exponents and amplitudes can be described by the 3d-Ising model is much smaller than that for binary mixtures of low molar mass samples.

(ii) Due to the relatively low viscosity of the PEG/PPG blend it was possible to achieve a thermodynamic equilibrium for the coexisting phases after one to two weeks. This allows to measure $\xi$ and $D$ separately for the PEG-rich and the PPG-rich phase. By comparing the critical amplitudes for the one phase region $\left(\xi_{0}\right)$ and for the two phase region $\left(\xi_{0, \mathrm{PEG}}{ }^{-}\right.$and $\left.\xi_{0, \mathrm{PPG}}{ }^{-}\right)$ the prediction for the amplitude ratio of $\xi_{0}^{-} / \xi_{0}=0.51$ (Ising model) was confirmed for the first time for a polymer/oligomer mixture.

\section{Acknowledgements}

The authors wish to thank Dr B. Steinhoff, L. Kühne, and Dr W. Mayer for the measurement of the cloud point curves of the mixtures. We also thank K. Rode and R. Ghahary for the characterisation of the molecular weight distributions and R. Fox for the measurements of the water contents of the samples. The financial support of the Deutsche Forschungsgemeinschaft (Grant No. Al 396/1-2) is gratefully acknowledged.

\section{References}

1 G. Meier, B. Momper and E. W. Fischer, J. Chem. Phys., 1992, 97, 5884 .

2 W. Theobald and G. Meier, Phys. Rev. E, 1995, 51, 5776.

3 H. Sato, N. Kuwahara and K. Kubota, Phys. Rev. E, 1996, 53, 3854. 
4 W. Mayer, S. Hoffmann, G. Meier and I. Alig, Phys. Rev. E, 1997, 55, 3102 .

5 P. Stepanek, T. P. Lodge, C. Kedrowski and F. S. Bates, J. Chem. Phys., 1991, 94, 8289.

6 R. Fernández, J. Fröhlich and A. D. Sokal, Random Walks, Critical Phenomena, and Triviality in Quantum Field Theory, Springer-Verlag, Berlin, 1992, Chap. 14.

7 G. Zalczer, A. Bourgou and D. Beysens, Phys. Rev. A, 1983, 28, 440 .

8 K. Hamano, S. Teshigawara, T. Koyama and N. Kuwahara, Phys. Rev. A, 1986, 33, 485.

9 J. Schmitz, L. Belkoura and D. Woermann, J. Chem. Phys., 1994, 101, 476.

10 J. Schmitz, Inaugural Dissertation, Universität Köln, Germany, 1994.

11 D. Schwahn, K. Mortensen and S. Janßen, Phys. Rev. Lett., 1994, 73, 1452.

12 B. Chu, F. J. Schoenes and M. E. Fisher, Phys. Rev., 1969, 185, 219.

13 S. Fusenig and D. Woermann, Ber. Bunsen-Ges. Phys. Chem., 1993, 97, 577.

14 J. P. Rakotoniaina, L. Belkoura and D. Woermann, Z. Phys. Chem., 1996, 196, 237.

15 P. G. de Gennes, J. Phys. Lett., 1977, 38, L441.

16 J. F. Joanny, J. Phys. A, 1978, 11, L117.

17 V. L. Ginzburg, Sov. Phys. Solid State, 1960, 2, 1824.

18 M. A. Anisimov, S. B. Kiselev, J. V. Sengers and S. Tang, Physica A, 1992, 188, 487.

19 S. Eckert, G. Meier and I. Alig, Phys. Chem. Chem. Phys., 2002, 4 3743 .
20 C. A. Tracy and B. M. McCoy, Phys. Rev. B, 1975, 12, 368.

21 H. B. Tarko and M. E. Fisher, Phys. Rev. B, 1975, 11, 1217.

2 H. Swinney and D. Henry, Phys. Rev A, 1973, 8, 2586.

3 D. W. Oxtoby and W. M. Gelbart, J. Chem. Phys., 1974, 61, 2957.

K. Kawasaki, Ann. Phys., 1970, 61, 1.

5 R. Folk and G. Moser, Phys. Rev. Lett., 1995, 75, 2706.

6 T. Ohta, J. Phys. C, 1977, 10, 791.

27 E. G. Siggia, B. I. Halperin and P. C. Hohenberg, Phys. Rev. B, $1976,13,2110$

28 G. B. Benedek, in Polarization, Matière et Rayonnement: vol. jubilaire en l'honneur d'Alfred Kastler, Presses universitaires de France, Paris, 1969.

29 K. Hamano, T. Kawazura, T. Koyama and N. Kuwahara, J. Chem. Phys., 1985, 82, 2718.

30 V. A. Rykov, Russ. J. Phys. Chem., 1985, 59, 464.

31 P. Schofield, Phys. Rev. Lett., 1969, 22, 606.

32 B. D. Josephson, J. Phys. Chem., 1969, 2, 1113.

33 K. Binder, J. Chem. Phys., 1983, 79, 6387.

34 B. Steinhoff, M. Rüllmann, L. Kühne and I. Alig, J. Chem. Phys., 1997, 107, 5217.

35 A. Z. Akcasu, in Dynamic light scattering, ed. W. Brown, Clarendon Press, Oxford, 1993.

36 J. C. LeGuillou and J. Zinn-Justin, Phys. Rev. B, 1980, 21, 3976; J. Zinn-Justin, Quantum Field Theory and Critical Phenonema, Oxford University Press, Oxford, 1989.

37 D. Schwahn, G. Meier, K. Mortensen and S. Janßen, J. Phys. II, 1994, 4, 837

38 J. V. Sengers, Int. J. Thermophys., 1985, 6, 203

39 S. M. Aharoni, Macromolecules, 1983, 16, 1722.

40 G. Heinrich, I. Alig and E. Donth, Polymer, 1988, 29, 1198. 